\title{
Adjusting the estimated proportion of breast cancer cases associated with BRCA1 and BRCA2 mutations: Public health implications
}

\author{
Monica R. McClain, PhD ${ }^{1}$, Glenn E. Palomaki, BS ${ }^{1}$, Katherine L. Nathanson, $\mathrm{MD}^{2}$, and James E. Haddow, $M D^{1}$
}

\begin{abstract}
Purpose: Mutations in BRCA1 or BRCA2 genes increase breast cancer risk. Assuring reliability of information about these mutations is increasingly important to the health care community; mutation testing is becoming more widespread. We describe a methodology for assessing such information. Methods: Our approach integrates four interdependent epidemiologic parameters: (1) the probability of developing breast cancer, (2) the proportion of breast cancer cases with a BRCA1 or BRCA2 mutation, (3) the proportion of women that carries a mutation, and (4) the proportion of women with a mutation that develops cancer. We assess the plausibility of estimates of these parameters from published reports and commonly accessed information sources. Results: Assuming a fixed probability of developing breast cancer, the following estimates for the other three epidemiologic parameters are derived for women by age $70: 1 \%$ to $2 \%$ of all breast cancer cases are associated with a BRCA1 or BRCA2 mutation; 1 in 300 to 1 in 465 women carry a mutation; and 35 to $65 \%$ of mutation carriers develop breast cancer. Within these ranges, however, only selected combinations are plausible. The proportion of mutation-related breast cancer is lower than listed in some common information sources (1 to $2 \%$ vs $6 \%$ ). Also, penetrance is somewhat lower and the carrier rate somewhat higher. Conclusions: The four epidemiologic parameters can be integrated to test their plausibility. BRCA1 and BRCA2 mutations are associated with only one-third as many breast cancer cases in the general population as reported by commonly accessed information sources. Genet Med 2005:7(1):28-33.
\end{abstract}

Key Words: breast cancer, genetics, public health, BRCA mutations.

Guidelines have been developed to aid clinicians in deciding when to offer BRCA1 and BRCA2 mutation testing. ${ }^{1-6}$ These guidelines recommend testing in specific circumstances, such as in women whose family history indicates an inherited predisposition to breast cancer. When a mutation is identified in an index case, cascade testing can be offered to other family members, thereby allowing primary prevention options to be considered. Implementation of BRCA1 and BRCA2 mutation testing by primary care providers has been sporadic and driven, in part, by direct-to-consumer advertising. ${ }^{7-11}$

At least two information sources about $B R C A 1$ and $B R C A 2$ mutation testing have been developed for use by primary care providers in the United States. ${ }^{12,13}$ In addition, two comprehensive reviews on $B R C A 1$ and $B R C A 2$ and hereditary breast cancer are available via internet access to health care providers, as well as the general public. ${ }^{14,15}$ The data summarized by these sources are not always complete and/or consistent, either in-

\footnotetext{
From the ${ }^{1}$ Foundation for Blood Research, Scarborough, Maine; and ${ }^{2}$ University of Pennsylvania, Department of Medicine, Philadelphia, Pennsylvania.

Monica R. McClain, PhD, Foundation for Blood Research, PO Box 190, 8 Nonesuch Road, Scarborough, $M E$.

Received: July 14, 2004.

Accepted: September 27, 2004.
}

DOI: 10.1097/01.GIM.0000151155.36470.FF ternally or with each other. These data are composed of three epidemiologic parameters: (1) the penetrance of BRCA1 and $B R C A 2$ mutations, (2) the mutation carrier rate in the general population, and (3) the proportion of all breast cancer cases that is associated with a mutation. Almost all estimates quoted for each of these epidemiologic parameters are indirectly derived in the original studies. For example, there have been no published studies where a large population-based sample has been selected and undergone mutation testing to determine the prevalence of mutation carriers (with the exception of individuals of Ashkenazi Jewish heritage ${ }^{16-21}$ ). Thus, this mutation carrier rate has been estimated indirectly. Similarly, an unbiased way to determine penetrance is a prospective study of individuals with and without mutations who are followed to see who does and does not develop breast cancer. Current penetrance estimates may be biased because they have been inferred by comparing family history in $B R C A$ mutation carriers to that in individuals without mutations, using women with breast cancer as the index case.

The present analysis is based on recognition that estimates for these three epidemiologic parameters, along with a fourth - the probability of developing breast cancer in the general population-are interdependent and that these relationships can be used to evaluate and refine the estimates. By integrating all four parameters, an initial evaluation indicates that 
one or more of the estimates from the information sources must be incorrect. To our knowledge, this is the first attempt to reconcile published estimates of these parameters in the context of a cohesive, integrated approach.

\section{MATERIALS AND METHODS}

The four epidemiologic parameters used in our integrated approach are described below and summarized in Table 1.

\section{(1) Probability of developing breast cancer (cumulative incidence)}

We first estimated the proportions of a cohort of one million 20-year-old women in the United States general population that will develop breast cancer: (1) by age 45 and (2) by age 70 . These were computed by the DevCan software, ${ }^{22}$ using the age-conditional probabilities by 5 -year intervals. ${ }^{23}$ Twenty years of age was selected as the reference point because $B R C A 1$ and BRCA2 mutation testing is recommended almost exclusively for adults and the incidence of breast cancer prior to age 20 is nearly zero. We selected 45 years as one cutoff because the studies on BRCA1 or BRCA2 mutations in early onset breast cancer enrolled women diagnosed between the ages of 35 and 55 years. ${ }^{24-28}$ We selected 70 years because it is the most com-

Table 1

Ranges of published values for four epidemiologic parameters that characterize the relationship between $B R C A 1$ and $B R C A 2$ mutations and breast cancer

\begin{tabular}{|c|c|c|}
\hline Epidemiologic parameter and definition & $\begin{array}{l}\text { By age } \\
45 \text { years }\end{array}$ & $\begin{array}{l}\text { By age } \\
70 \text { years }\end{array}$ \\
\hline Cumulative incidence (\%) & 1.19 & 9.67 \\
\hline \multicolumn{3}{|l|}{$\begin{array}{l}\text { Proportion of women in the general } \\
\text { population that will develop breast } \\
\text { cancer by a given age } \text { en }^{22}\end{array}$} \\
\hline Clinical sensitivity (\%) & $5.4-12.6^{24-28}$ & $2-10^{28-3}$ \\
\hline \multicolumn{3}{|c|}{$\begin{array}{l}\text { Proportion of women with breast cancer in } \\
\text { the general population that has a } B R C A 1 \\
\text { or } B R C A 2 \text { mutation by a given age }\end{array}$} \\
\hline \multicolumn{3}{|l|}{ Penetrance (\%) } \\
\hline \multicolumn{3}{|c|}{$\begin{array}{l}\text { Proportion of women with a } B R C A 1 \text { or } \\
B R C A 2 \text { mutation that develops breast } \\
\text { cancer by a given age }\end{array}$} \\
\hline$B R C A 1$ alone $e^{25,35-39,41}$ & $20-40$ & $35-85$ \\
\hline$B R C A 2$ alone $25,35-37,41,43$ & $10-25$ & $45-85$ \\
\hline$B R C A 1$ and $B R C A 2^{25,35-37}$ & $15-30^{a}$ & $35-65^{a}$ \\
\hline \multicolumn{3}{|l|}{ Carrier rate } \\
\hline \multicolumn{3}{|c|}{$\begin{array}{c}\text { The rate of women with a } B R C A 1 \text { or } B R C A 2 \\
\text { mutation in the general population }\end{array}$} \\
\hline$B R C A 1$ alone $25,28,32,36,37$ & \multicolumn{2}{|c|}{1 in 800 to 1 in 1,400} \\
\hline$B R C A 2$ alone e $^{25,28,36,37}$ & \multicolumn{2}{|c|}{1 in 450 to 1 in 800} \\
\hline$B R C A 1$ and $B R C A 2^{25,28,31,36,37}$ & \multicolumn{2}{|c|}{1 in 300 to 1 in 450} \\
\hline
\end{tabular}

${ }^{a}$ Approximate weighted average of the penetrance for BRCA1 and BRCA2 from population based studies. mon age cutoff used in studies reporting the proportion of women with a mutation that develops breast cancer.

\section{(2) Proportion of women with breast cancer that has a BRCA1 or BRCA2 mutation (clinical sensitivity)}

Direct estimates have been made for the proportion of earlyonset breast cancer with these mutations. ${ }^{24-28}$ The adjusted range of these direct estimates is reported in Table 1. The details of how adjustments were made to these estimates are shown in the Appendix. These conservative adjustments account for deleterious mutations that have been missed because the studies used methodologies that are less sensitive than the gold standard of direct DNA sequencing. The adjustments do not account for mutations that are not detectable by sequencing. The estimates of clinical sensitivity are heterogeneous. Therefore, rather than calculating a summary estimate, we have provided a sensitivity analysis over the range of published estimates in our integrated approach. Sensitivity analysis is appropriate when there is not consensus around what value should be assigned to a given parameter.

The proportion of breast cancer cases by age 70 associated with $B R C A 1$ mutations is derived from both direct ${ }^{29,30}$ and indirect ${ }^{31,32}$ estimates, whereas only indirect estimates have been reported for the contribution of mutations in both BRCA1 and BRCA2 genes to breast cancer. ${ }^{28,33,34}$ The range of these estimates is also shown in Table 1. We include a sensitivity analysis for this estimate, as well, in our integrated approach.

\section{(3) Proportion of women with a BRCA1 or BRCA2 mutation that develops breast cancer (penetrance)}

Estimates have been reported in both population-based individuals and high risk families. ${ }^{25,35-44}$ Table 1 shows that these point estimates cover a broad range. The corresponding confidence intervals are also wide, indicating that the estimates are not precise. Biases are inherent in the study designs used to produce these estimates. ${ }^{45}$ For these reasons, we also include a sensitivity analysis for this variable in our integrated approach. In our analysis, separate mutation penetrance estimates are used for the two age cutoffs. By age 45, the penetrance of $B R C A 1$ mutations is higher than for BRCA2 mutations, but only 40 out of every 100 mutation carriers will have a BRCA1 mutation, based on the estimated prevalence of mutations (see no. 4). For this age cutoff, our integrated approach utilizes a weighted average of the population-based penetrance estimates. There is no consensus among the studies on the BRCA1 and $B R C A 2$ mutation penetrance estimates for breast cancer by age 70 . Of the three population-based studies that provide penetrance estimates for mutations in both genes in the nonAshkenazi Jewish population, two report higher estimates for $B R C A 2$ mutations ${ }^{25,36}$ and one reports a lower estimate. ${ }^{35}$ For our analysis, the penetrance estimates for $B R C A 1$ and $B R C A 2$ mutations are assumed to be the same for breast cancer by the age of 70 . 


\section{(4) Proportion of women with BRCA1 and BRCA2 mutations in the general population (carrier rate)}

This proportion has been estimated indirectly by several studies $^{25,28,31,32,36,37}$ and can be expressed as a carrier rate (e.g., $0.2 \%=1$ in 500). Table 1 shows the range of these estimates.

The relationship among the four epidemiologic parameters is based on Bayes theorem and is defined by our integrated approach as:

$$
\text { carrier rate }(1 \text { in } N)=\frac{1}{(\mathrm{ci} \times \mathrm{cs}) / \mathrm{p}}
$$

where $\mathrm{ci}=$ cumulative incidence of breast cancer (\%) by a given age, $\mathrm{cs}=$ clinical sensitivity of $B R C A 1 / 2$ mutations $(\%)$, and $\mathrm{p}=$ penetrance of $B R C A 1 / 2$ mutations (\%). Any three of these epidemiologic parameter estimates can be used to compute the fourth. This integrated approach is then applied to data from original research studies and to commonly accessed information sources that provide a review of these data.

\section{RESULTS}

Table 2 shows a two-way sensitivity analysis for penetrance of BRCA1 and BRCA2 mutations and the proportion of breast cancer cases with a mutation. The top half of the table uses the cumulative incidence of cancer in the general U.S. population by age 45 years (1.19\%), whereas the bottom half uses the cumulative incidence by age 70 years $(9.67 \%)$. The upper and lower limits for proportions of breast cancer cases with a mutation (columns) and penetrance (rows) are taken from Table 1. The associated carrier rates (table entries) are then computed for each combination. For example, by age 45 years, with mutations present in 5\% of cancer cases and a penetrance of $25 \%$, the carrier rate is computed to be 1 in 420 women (boxed entry in top half of Table 1). Fig. 1 shows how these calculations are made. As the proportion of cancers associated with a mutation increases, the carrier rate must also increase. However, as the penetrance increases, the carrier rate must decrease.

In order to focus on plausible combinations of three of the epidemiologic parameters, we have bolded selected entries in Table 2 when the four parameters are internally consistent (e.g., the computed carrier rate based on the other parameters is within the range of published carrier rates specified in Table 1). Columns showing the proportion of breast cancer cases associated with mutations of $9 \%$ or higher are not shown, because none of the corresponding carrier rates are within the range specified. According to this analysis, between $4 \%$ and $8 \%$ of breast cancer cases by age 45 occur in women with a BRCA1 or BRCA2 mutation.

In the bottom half of Table 2, the range of carrier rates validated for the 45 year age group is used to help select reasonable and consistent combinations of penetrance and proportions of cancers by age 70 that are associated with mutations. The range of penetrance estimates is again taken from Table 1. Entries are bolded, if they are internally consistent. When the proportion of breast cancer cases associated with mutations exceeds $2 \%$, none of the corresponding carrier rates are within the range specified in Table 1. According to this integrated approach, the proportion of women with breast cancer by age 70 that has a $B R C A 1$ or BRCA 2 mutation is between 1 and $2 \%$.

The top half of Table 3 compares the epidemiologic parameter estimates reported by the four information sources commonly used by health care providers and patients. Only one of these sources cites estimates for all four of the epidemiologic parameters. ${ }^{12}$ The bottom half of Table 3 shows estimates derived using our integrated approach for all four epidemiologic

Table 2

$B R C A 1$ and $B R C A 2$ mutation carrier rates computed using the interrelationships among the cumulative incidence of breast cancer, the proportion of women with breast cancer that has a mutation, and the penetrance of mutations

Breast cancer by age 45 years (cumulative incidence $=1.19 \%$ )

Proportion of Women with Breast Cancer that has a BRCA1 or BRCA2 Mutation

\begin{tabular}{|c|c|c|c|c|c|}
\hline \multirow[b]{2}{*}{ Penetrance (\%) } & & & & & \\
\hline & $4 \%$ & $5 \%$ & $6 \%$ & $7 \%$ & $8 \%$ \\
\hline 15 & 1 in 315 & 1 in 250 & 1 in 210 & 1 in 180 & 1 in 160 \\
\hline 20 & 1 in 420 & 1 in 340 & 1 in 280 & 1 in 240 & 1 in 210 \\
\hline 25 & 1 in 525 & 1 in 420 & 1 in 350 & 1 in 300 & 1 in 260 \\
\hline 30 & 1 in 630 & 1 in 505 & 1 in 420 & 1 in 360 & 1 in 315 \\
\hline
\end{tabular}

Breast cancer by age 70 years (cumulative incidence $=9.67 \%$ )

Proportion of Women with Breast Cancer that has a BRCA1 or BRCA2 Mutation

\begin{tabular}{|c|c|c|c|c|c|}
\hline \multirow[b]{2}{*}{ Penetrance (\%) } & \\
\hline & $1.00 \%$ & $1.25 \%$ & $1.50 \%$ & $1.75 \%$ & $2.00 \%$ \\
\hline 35 & 1 in 360 & 1 in 290 & 1 in 240 & 1 in 205 & 1 in 180 \\
\hline 45 & 1 in 465 & 1 in 370 & 1 in 310 & 1 in 265 & 1 in 235 \\
\hline 55 & 1 in 570 & 1 in 455 & 1 in 380 & 1 in 325 & 1 in 285 \\
\hline 65 & 1 in 670 & 1 in 540 & 1 in 450 & 1 in 395 & 1 in 335 \\
\hline
\end{tabular}

Bolded items represent plausible combinations of the four epidemiologic parameter estimates and boxed item represents the combination used in Fig. 1 (see text). 


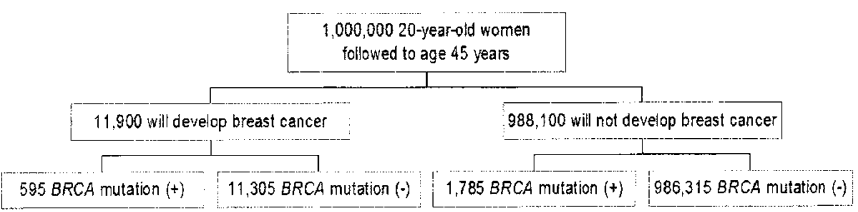

Carrier rate: $2,380 / 1,000,000$ or 1 in 420

Fig. 1. Flowchart showing an example of how the cumulative incidence of breast cancer and the three epidemiologic parameters are related. In this example, a hypothetical cohort of one million women is followed from age 20 to age 45 . Overall, 11,900 cases of breast cancer are expected (cumulative incidence). Among these 11,900 women with breast cancer, $595(5 \%)$ will have a BRCA1 or BRCA2 mutation (clinical sensitivity). When the penetrance estimate of $25 \%$ is applied, 2,380 women carry a mutation ( 595 / 0.25$)$. Thus, 1,785 women with a mutation have not developed breast cancer by age $45(2,380-595)$. The carrier rate is, therefore, 1 in 420. Numbers in the remaining boxes in Fig. 1 are computed by subtraction.

parameters when the other three parameters are set to the values in the top half of Table 3. The carrier rate is computed to be between 1 in 133 and 1 in 207 when the cumulative incidence of breast cancer is $7 \%$, the proportion of cancer cases with a mutation is $6 \%$ and penetrance is allowed to vary from 56 to $87 \%$. The proportion of cancer cases with a mutation is computed to be between 1.6 and $2.5 \%$ when the cumulative incidence of breast cancer is 7\%, the carrier rate is 1 in 500 and the penetrance is allowed to vary between $56 \%$ and $87 \%$. The penetrance is computed to exceed $100 \%$ (not plausible) when the cumulative incidence of breast cancer is $7 \%$, the proportion of cancer cases with a mutation is $6 \%$ and the carrier rate is 1 in 500 .

These computations demonstrate that epidemiologic estimates from one of the four information sources are not internally consistent. ${ }^{12}$ Neither the computed carrier rate nor the computed penetrance are plausible, indicating that the proportion of cancer cases with a mutation in the top half of the table must be overestimated. Although the reported value of $6 \%$ is in the middle of the published range of $2 \%$ to $10 \%$ (Table 1 ), our analysis shows that the most plausible value is between $1 \%$ and $2 \%$.

\section{DISCUSSION}

We developed the integrated approach described above to reconcile individual estimates that each have varying degrees of reliability. Our study confirms published estimates showing a combined carrier rate for BRCA1 and BRCA2 mutations in the general population of approximately 1 in 400 . For women up to age 45 , the mutation penetrance also falls within the range reported by published population-based studies, but as expected, it is lower than the reported estimates in high-risk families. Also, the proportion of women with breast cancer by age 45 that carries a mutation is between $4 \%$ and $8 \%$, near the lower end of the published range. For women with breast cancer by age 70 , the proportion with a $B R C A 1$ or $B R C A 2$ mutation is between $1 \%$ and $2 \%$. This estimate is similar to the two studies that directly measured clinical sensitivity of BRCA1 mutations, ${ }^{29,30}$ but is lower than would be expected had BRCA2 mutations been identified, as well. The estimate of $1 \%$ to $2 \%$ is just below the lower end of the range in the published literature that indirectly estimated clinical sensitivity and considerably below the estimates provided by some of the most commonly available information sources. The studies that indirectly estimated the clinical sensitivity were all published within 5 years of the cloning of BRCA1.28,31-34 The estimates derived from these studies may be higher due to the indirect methods used and/or the limited data available on BRCA1 and $B R C A 2$ mutations at that time. The clinical sensitivity estimate that is reported by some of the common information sources $^{12,13}$ references an early study that estimated the contribution of BRCA1 and BRCA2 mutations to breast cancer in families with at least four cases of breast cancer. ${ }^{41}$ This estimate is higher than those derived from cancer cases in the general population.

Studies that are composed mainly of Ashkenazi Jewish women are not included in our analysis, because at least one epidemiologic parameter (mutation carrier rate) is higher by about a factor of 10. Also, the cumulative incidence of breast cancer is less well defined in this group. These factors indicate that this group of women should be considered separately. The studies that provided estimates for the epidemiologic parameters used in our integrated approach included primarily Caucasian women in the United States or Western Europe. Therefore, analyses involving other ethnic/racial groups may yield different results.

Our approach can be applied, however, to other groups (e.g., Ashkenazi Jewish women), taking into account special considerations such as the important impact of founder mutations on the carrier rate and, perhaps, genotype/phenotype relationships. Another application might involve BRCA1 and $B R C A 2$ mutation testing in the presence of a family history of breast and/or ovarian cancer combined. An extended approach could be created that addresses such mutation testing for breast and/or ovarian cancer. This approach might also be adapted to other disorders with a genetic component.

There are several limitations to our integrated approach. First, the penetrance estimates for BRCA1 and BRCA2 mutations are combined. There are substantial differences in penetrance for early onset breast cancer between mutations in each gene that we address by utilizing a weighted average. It is not yet clear if there are differences in penetrance for breast cancer by age 70 . We assume that the penetrance is the same for mutations in BRCA1 and BRCA2. However, even if the penetrance estimate is $65 \%$ higher for mutations in one gene compared with the other, the impact on clinical sensitivity is $<1 \%$. Another approach could be to consider mutations in each gene separately. Furthermore, there may be variations in penetrance by mutation type (e.g., frameshift, nonsense) or mutation position (e.g., ovarian cancer cluster region). ${ }^{41,46-49}$ Second, the use of Surveillance, Epidemiology, and End Results data in the DevCan software is the only estimate of breast cancer incidence in the U.S. that is generalizeable to the general population. The majority of studies that estimate mutation pen- 
Table 3

Breast cancer and $B R C A 1 / B R C A 2$ mutations for women up to age 70 years: A comparison among commonly used information sources

\begin{tabular}{|c|c|c|c|c|}
\hline Epidemiologic parameter & Myriad $^{12}$ & $\begin{array}{c}\text { American } \\
\text { Medical } \\
\text { Association }^{13}\end{array}$ & $\begin{array}{c}\text { Physician } \\
\text { data query }\end{array}$ & Gene reviews ${ }^{15}$ \\
\hline Cumulative incidence (\%) & 7 & Not given & Not given & Not given \\
\hline Cancer cases with a mutation (\%) & 6 & 6 & 2 & Not given \\
\hline Penetrance of mutations (\%) & $56-87$ & $56-87$ & $36-85$ & $36-86$ \\
\hline Carrier rate $(1$ in $n)$ & 500 & Not given & 500 & $500-1000$ \\
\hline \multicolumn{5}{|l|}{ Calculated from above $^{a}$} \\
\hline Cancer cases with a mutation (\%) & $1.6-2.5$ & Insufficient & Insufficient & Insufficient \\
\hline Penetrance of mutations (\%) & $>100$ & Information & Information & Information \\
\hline Carrier rate $(1$ in $n)$ & $133-207$ & & & \\
\hline Internally consistent & No & Unknown & Unknown & Unknown \\
\hline
\end{tabular}

${ }^{a}$ Each epidemiologic parameter is calculated by setting the other three parameters listed by the same information source as constants in our integrated approach.

etrance have used age-specific breast cancer incidence in England and Wales..$^{35-37,41}$ Because the incidence of breast cancer is lower in these countries, the resulting penetrance estimates may not be directly applicable to the U.S. population.

This study highlights the need for accurate and complete information regarding the burden of $B R C A 1$ and $B R C A 2 \mathrm{mu}-$ tations on breast cancer in the U.S. Only one of the four commonly accessed information sources examined in this study contains estimates for the four epidemiologic parameters. ${ }^{12}$ The remaining three information sources report estimates for two $\mathrm{O}^{13,15}$ or three $^{14}$ of the epidemiologic parameters. The quality of the evidence cited in support for these estimates is questionable and, in some cases, references are absent. For example, the mutation carrier rate is not cited in one information source. ${ }^{13}$ It is cited to be between 1 in 500 and 1 in 1000 with no references by another source. ${ }^{15}$ The third information source cites the mutation carrier rate to be 1 in 800 for $B R C A 1$ mutations (no reference) and 1 in 500 for all mutations but the reference given does not provide a mutation carrier rate estimate. ${ }^{14}$ The fourth information source cites a 1 in 500 mutation carrier rate. However, the given reference is not the original data source and only quotes this carrier rate for BRCA1 mutations. ${ }^{12}$ None of the four information sources cite the research articles from which the mutation carrier rate is inferred, which we have summarized in this study.

Our study finds that $B R C A 1$ and $B R C A 2$ mutations appear to account for only one-third as many breast cancer cases by age 70 as reported in the two information sources for physicians. From the viewpoint of public health planning, the downward revision in this estimate means that the potential reduction in breast cancer cases resulting from mutation testing in the general population is likely to be substantially lower than generally thought.

\section{APPENDIX}

Direct sequencing is expected to have the highest clinical sensitivity of all mutation detection methods. One study used direct sequencing of $B R C A 1$ as the gold standard and evaluated the performance of SSCP, CSGE, two-dimensional gene scanning (TDGS), and denaturing high performance liquid chromatography (dHPLC). These four methods detected 65\%, $60 \%, 91 \%$, and $100 \%$ of the mutations detected by sequencing, respectively..$^{50} \mathrm{~A}$ second study that again used direct sequencing as the gold standard found that mutation analysis by SSCP accounted for $94 \%$ of the detected variants, whereas dHPLC resolved $100 \%$ of the alterations..$^{51}$ We used conservative estimates of mutation detection to adjust the clinical sensitivities of mutation testing in women with early onset breast cancer (Table 4).

\section{ACKNOWLEDGMENTS}

Support for this study was provided by a cooperative agreement (UR3/CCU319352) with the Centers for Disease Control and Prevention, Office of Genomics and Disease Prevention. We thank Muin Khoury, MD, PhD, Paula Yoon, ScD, MPH, and Linda A. Bradley, PhD, Office of Genomics and Disease Prevention, Centers for Disease Control and Prevention for

Table 4

Adjustments for potential methodological underascertainment made to clinical sensitivity estimates of $B R C A 1 / 2$ mutation testing

\begin{tabular}{|c|c|c|}
\hline Reference & $B R C A 1 / 2$ methodology & $\begin{array}{c}\text { Estimated } \\
\text { proportion } \\
\text { of mutations } \\
\text { detected }(\%)\end{array}$ \\
\hline Malone et al. ${ }^{27}$ & SSCP & 65 \\
\hline Loman et al. ${ }^{26}$ & PTT/SSCP/dHPLC/direct sequence & 80 \\
\hline Hopper et al. ${ }^{24}$ & PTT/HA & 85 \\
\hline Peto et al. ${ }^{28}$ & CSGE & 60 \\
\hline $\begin{array}{l}\text { Anglian Breast Cancer } \\
\text { Study Group }{ }^{25}\end{array}$ & MHA & 60 \\
\hline
\end{tabular}


their support of the ACCE project. We also thank Ingrid Hall, $\mathrm{PhD}$, Division of Cancer Prevention and Control, National Center for Chronic Disease Prevention and Health Promotion, Centers for Disease Control and Prevention for helping support the project.

\section{References}

1. Genetic susceptibility to breast and ovarian cancer: assessment, counseling and testing guidelines. American College of Medical Genetics Foundation. Available at: http://www.health.state.ny.us/nysdoh/cancer/obcancer/contents.htm. Accessed September 10, 2004.

2. Advice about familial aspects of breast cancer and ovarian cancer: A guide for health professionals: National Health and Medical Research Council, National Breast Cancer Centre; 2000. Available at: http://www.nbcc.org.au. Accessed September 10, 2004.

3. Elwyn G, Iredale R, Gray J. Reactions of GPs to a triage-controlled referral system for cancer genetics. Fam Pract 2002;19:65-71.

4. Lucassen A, Watson E, Harcourt J et al. Guidelines for referral to a regional genetics service: GPs respond by referring more appropriate cases. Fam Pract 2001;18:135-140.

5. American Society of Clinical Oncology policy statement update: Genetic testing for cancer susceptibility. J Clin Oncol 2003;11:11.

6. Genetic/familial high-risk assessment: breast and ovarian. Jenkintown, Pa: National Comprehensive Cancer Network; 2003. Available at: http://www.nccn.org. Accessed September 10, 2004.

7. Rose PW, Watson E, Yudkin P et al. Referral of patients with a family history of breast/ ovarian cancer-GPs' knowledge and expectations. Fam Pract 2001;18:487-490.

8. Wideroff L, Freedman AN, Olson L et al. Physician use of genetic testing for cancer susceptibility: results of a national survey. Cancer Epidemiol Biomarkers Prev 2003; 12:295-303.

9. Sifri R, Myers R, Hyslop T et al. Use of cancer susceptibility testing among primary care physicians. Clin Genet 2003;64:355-360.

10. Mouchawar J, Klein CE, Mullineaux L. Colorado family physicians' knowledge of hereditary breast cancer and related practice. J Cancer Educ Spring 2001;16:33-37.

11. Friedman LC, Cooper HP, Webb JA, Weinberg AD, Plon SE. Primary care physicians' attitudes and practices regarding cancer genetics: a comparison of 2001 with 1996 survey results. J Cancer Educ 2003;18:91-94.

12. Hereditary risk of breast and ovarian cancer. A professional and community education program. Salt Lake City: Myriad Genetic Laboratories; 2001. Available at: http://www.myriad.com. Accessed September 10, 2004.

13. Identifying and managing hereditary risk for breast and ovarian cancer. Chicago, Ill: American Medical Association; 2001. Available at: http://www.ama-assn.org. Accessed September 10, 2004.

14. Genetics of breast and ovarian cancer (PDQ). National Cancer Institute. 6/10/04 Available at: http://www.cancer.gov/cancerinfo/pdq/genetics/breast-and-ovarian. Accessed September 10, 2004.

15. Petrucelli MS, Daly MB, Burke W et al. BRCA1 and BRCA2 hereditary breast/ovarian cancer. 09/03/04. Available at: http://www.genetests.org. Accessed September 10, 2004

16. Fodor FH, Weston A, Bleiweiss IJ et al. Frequency and carrier risk associated with common BRCA1 and BRCA2 mutations in Ashkenazi Jewish breast cancer patients. Am J Hum Genet 1998;63:45-51.

17. Hartge P, Struewing JP, Wacholder S, Brody LC, Tucker MA. The prevalence of common BRCA1 and BRCA2 mutations among Ashkenazi Jews. Am J Hum Genet 1999;64:963-970.

18. Oddoux C, Struewing JP, Clayton CM et al. The carrier frequency of the BRCA2 $6174 \mathrm{delT}$ mutation among Ashkenazi Jewish individuals is approximately $1 \%$. Nat Genet 1996;14:188-190.

19. Roa BB, Boyd AA, Volcik K, Richards CS. Ashkenazi Jewish population frequencies for common mutations in BRCA1 and BRCA2. Nat Genet 1996;14:185-187.

20. Struewing JP, Abeliovich D, Peretz T et al. The carrier frequency of the BRCA 185delAG mutation is approximately 1 percent in Ashkenazi Jewish individuals. Nat Genet Oct 1995;11:198-200.

21. Struewing JP, Hartge $P$, Wacholder $S$ et al. The risk of cancer associated with specific mutations of BRCA1 and BRCA2 among Ashkenazi Jews. N Engl J Med May 15 1997;336:1401-1408.

22. DevCan software: Probability of developing or dying of cancer [computer program] Version 5.1: Statistical Research and Application Branch, National Cancer Institute; 2003. Available at: http://srab.cancer.gov/devcan. Accessed September 10, 2004.

23. Fay MP, Pfeiffer R, Cronin KA, Le C, Feuer EJ. Age-conditional probabilities of developing cancer. Stat Med 2003;22:1837-1848.

24. Hopper JL, Southey MC, Dite GS et al. Population-based estimate of the average age-specific cumulative risk of breast cancer for a defined set of protein-truncating mutations in BRCA1 and BRCA2. Australian Breast Cancer Family Study. Cancer Epidemiol Biomarkers Prev 1999;8:741-747.
25. Prevalence and penetrance of BRCA1 and BRCA2 mutations in a population-based series of breast cancer cases. Anglian Breast Cancer Study Group. Br J Cancer. 2000; 83:1301-1308.

26. Loman N, Johannsson O, Kristoffersson U, Olsson H, Borg A. Family history of breast and ovarian cancers and BRCA1 and BRCA2 mutations in a populationbased series of early-onset breast cancer. J Natl Cancer Inst 2001;93:1215-1223.

27. Malone KE, Daling JR, Neal C et al. Frequency of BRCA1/BRCA2 mutations in a population-based sample of young breast carcinoma cases. Cancer 2000;88:1393-1402.

28. Peto J, Collins N, Barfoot R et al. Prevalence of BRCA1 and BRCA2 gene mutations in patients with early-onset breast cancer. J Natl Cancer Inst 1999;91:943-949.

29. Newman B, Mu H, Butler LM, Millikan RC, Moorman PG, King MC. Frequency of breast cancer attributable to BRCAl in a population-based series of American women. JAMA 1998;279:915-921.

30. Anton-Culver H, Cohen PF, Gildea ME, Ziogas A. Characteristics of BRCA1 mutations in a population-based case series of breast and ovarian cancer. Eur J Cancer 2000;36:1200-1208.

31. Whittemore AS, Gong G, Itnyre J. Prevalence and contribution of BRCA1 mutations in breast cancer and ovarian cancer: results from three U.S. population-based casecontrol studies of ovarian cancer. Am J Hum Genet 1997;60:496-504.

32. Ford D, Easton DF, Peto J. Estimates of the gene frequency of BRCAl and its contribution to breast and ovarian cancer incidence. Am J Hum Genet 1995;57:1457-1462.

33. Claus EB, Schildkraut JM, Thompson WD, Risch NJ. The genetic attributable risk of breast and ovarian cancer. Cancer 1996;77:2318-2324.

34. Newman B, Millikan RC, King MC. Genetic epidemiology of breast and ovarian cancers. Epidemiol Rev 1997;19:69-79.

35. Antoniou A, Pharoah PD, Narod S et al. Average risks of breast and ovarian cance associated with BRCA1 or BRCA2 mutations detected in case series unselected for family history: a combined analysis of 22 studies. Am J Hum Genet 2003;72:1117-1130.

36. Antoniou AC, Pharoah PD, McMullan $\mathrm{G}$ et al. A comprehensive model for familial breast cancer incorporating BRCA1, BRCA2 and other genes. Br J Cancer 2002;86:76-83.

37. Antoniou AC, Gayther SA, Stratton JF, Ponder BA, Easton DF. Risk models for familial ovarian and breast cancer. Genet Epidemiol 2000;18:173-190.

38. Brose MS, Rebbeck TR, Calzone KA, Stopfer JE, Nathanson KL, Weber BL. Cance risk estimates for BRCA1 mutation carriers identified in a risk evaluation program. J Natl Cancer Inst 2002;94:1365-1372.

39. Easton DF, Ford D, Bishop DT. Breast and ovarian cancer incidence in BRCA1mutation carriers. Breast Cancer Linkage Consortium. Am J Hum Genet 1995;56: 265-271.

40. Ford D, Easton DF, Bishop DT, Narod SA, Goldgar DE. Risks of cancer in BRCA1 mutation carriers. Breast Cancer Linkage Consortium. Lancet 1994;343:692-695.

41. Ford D, Easton DF, Stratton $\mathrm{M}$ et al. Genetic heterogeneity and penetrance analysis of the BRCA1 and BRCA2 genes in breast cancer families. The Breast Cancer Linkage Consortium. Am J Hum Genet 1998;62:676-689.

42. Risch HA, McLaughlin JR, Cole DE et al. Prevalence and penetrance of germline BRCA1 and BRCA2 mutations in a population series of 649 women with ovarian cancer. Am J Hum Genet 2001;68:700-710.

43. Schubert EL, Lee MK, Mefford HC et al. BRCA2 in American families with four or more cases of breast or ovarian cancer: recurrent and novel mutations, variable expression, penetrance, and the possibility of families whose cancer is not attributable to BRCA1 or BRCA2. Am J Hum Genet 1997;60:1031-1040.

44. Scott CL, Jenkins MA, Southey MC et al. Average age-specific cumulative risk of breast cancer according to type and site of germline mutations in BRCAl and BRCA2 estimated from multiple-case breast cancer families attending Australian family cancer clinics. Hum Genet 2003;112:542-551.

45. Begg CB. On the use of familial aggregation in population-based case probands for calculating penetrance. J Natl Cancer Inst 2002;94:1221-1226.

46. Gayther SA, Mangion J, Russell P et al. Variation of risks of breast and ovarian cancer associated with different germline mutations of the BRCA2 gene. Nat Genet 1997; 15:103-105.

47. Neuhausen SL, Godwin AK, Gershoni-Baruch R et al. Haplotype and phenotype analysis of nine recurrent BRCA2 mutations in 111 families: results of an international study. Am J Hum Genet 1998;62:1381-1388.

48. Thompson D, Easton D. Variation in cancer risks, by mutation position, in BRCA2 mutation carriers. Am J Hum Genet 2001;68:410-419.

49. Thompson D, Easton D. Variation in BRCAl cancer risks by mutation position. Cancer Epidemiol Biomarkers Prev 2002;11:329-336.

50. Eng C, Brody LC, Wagner TM et al. Interpreting epidemiological research: blinded comparison of methods used to estimate the prevalence of inherited mutations in BRCA1. J Med Genet 2001;38:824-833.

51. Gross E, Arnold N, Goette J, Schwarz-Boeger U, Kiechle M. A comparison of BRCA1 mutation analysis by direct sequencing, SSCP and DHPLC. Hum Genet 1999;105: $72-78$. 